\title{
Planetary Exploration and Archaeology: Heritage Conservation
}

\author{
John B. Campbell \\ School of Anthropology, Archaeology and Sociology, James Cook \\ University, Cairns, Australia
}

Planetary exploration is resulting in the creation of new archaeological sites, material and debris on planets and their moons, and in various orbits round the Earth, Mars, the Sun etc. The main off-Earth bodies with sites so far are the Moon and Mars. Although thousands of archaeological sites on Earth are protected for their heritage value, no sites off-Earth are properly protected as yet. Sites off-Earth need to be ranked for their comparative heritage significance and protocols developed for the conservation and protection of the more significant sites and artifacts, before specimens are collected and returned to Earth in an uncontrolled (from heritage points of view) manner. A new United Nations Space Heritage Treaty is needed, or at least appropriate IAU and WAC (World Archaeological Congress) protocols agreed by the various parties concerned. The UN Outer Space Treaty 1967 is very out of date and a product of the Cold War.

The current archaeology of the Moon faces an urgent need for assessment of heritage and future scientific values of the Apollo landing sites. As the landing site where people set foot on another world for the first time, Apollo 11's Tranquillity Base is of high international significance and worthy of the equivalent of World Heritage status. Other NASA mission sites should also be considered for assessment, as should the landing and crash sites of the former Soviet Union. Future ESA, Japanese, Chinese, Indian, Russian and NASA sites should be recorded (at least remotely) as they are formed; this would not be that different in principle from ethnoarchaeology on Earth (i.e. the archaeology of the physical activities, material creations and discarded materials of living people). There is ample material on the Moon for landscape archaeology.

The archaeology of Mars would focus initially on assessment of NASA's Viking 1, Viking 2 and Pathfinder landers, as well as various crash sites such as the former USSR's Mars 2 (1971) and NASA's Mars Polar Lander (1999), once they are relocated. Monitoring selected sites and artifacts on Mars would be of particular interest as abrasion and cryoclastic action will almost certainly be occurring. A register of sites of heritage significance should be established before Mars is colonized or equipment is sampled by private corporations as is now intended for some of the Apollo sites on the Moon. The current missions to Mars (ESA's Mars Express with its Beagle 2 lander, NASA's Mars Exploration Rovers Spirit and Opportunity landers and Japan's Nozomi) will have varying levels of heritage significance, some of which will depend on their respective scientific successes (mapping, relocation of Mars Polar Lander, detection of evidence for past or present extremophiles etc.).

Conservation of sites on Venus would be impracticable as any surviving American (NASA) or Russian (USSR) artifacts will be very corroded. However, 
investigation of rates of corrosion and variations in resistance according to kind of material and position in the Venusians landscape would be of more than academic interest in planning future surface missions. Planned missions to the inner planets such as ESA's Venus Express and the Mercury mission, BepiColombo, should be judged and monitored for their heritage significance as they unfold.

A future detailed register of sites on asteroids (such as Eros), Jovian moons (e.g. Europa), saturnian moons (e.g. Titan) and further out (e.g. Pluto and Charon) would be very useful to establish and maintain as they are created. Discovery of extremophiles or other life on any or all of Europa, Titan or Mars will present other ethical challenges. In general, a well structured and constantly updated, internationally mirrored heritage database on missions, orbits, trajectories, probes, landing sites, crash sites, scientific significance, national significance, international significance, interplanetary significance (stage of exploration of Solar System), conservation status, threats to conservation, protection status, expected costs of preservation, actual costs of preservation etc. will be essential for the archaeology of the Solar System. Unlike what has often happened on Earth, being ready with a plan, knowledge of the site/artifact, likely costs of intervention etc., would mean that appropriate action could be decided and carried out before significant sites/artifacts are damaged or removed by either looting or uncontrolled collecting. 